\title{
A Response to: Letter to the Editor Regarding Management of Adult Patients with COVID-19 Outside Intensive Care Units: Guidelines from the Italian Society of Anti-Infective Therapy (SITA) and the Italian Society of Pulmonology (SIP)
}

\begin{abstract}
Matteo Bassetti • Daniele Roberto Giacobbe (D) - Paolo Bruzzi • Emanuela Barisione • Stefano Centanni •
Nadia Castaldo • Silvia Corcione • Francesco Giuseppe De Rosa • Fabiano Di Marco • Andrea Gori • Andrea Gramegna • Guido Granata • Angelo Gratarola • Alberto Enrico Maraolo • Malgorzata Mikulska •

Andrea Lombardi · Federico Pea $\cdot$ Nicola Petrosillo $\cdot$ Dejan Radovanovic $\cdot$ Pierachille Santus $\cdot$ Alessio Signori Emanuela Sozio · Elena Tagliabue - Carlo Tascini • Carlo Vancheri • Antonio Vena • Pierluigi Viale • Francesco Blasi on behalf of the Italian Society of Anti-infective Therapy (SITA), the Italian Society of Pulmonology (SIP)
\end{abstract}

Received: October 15, 2021 / Accepted: November 9, 2021 / Published online: December 10, 2021

(c) The Author(s) 2021

Keywords: COVID-19; mAbs; Monoclonal antibodies; SARS-CoV-2; Casirivimab; Imdevimab; Guidelines

M. Bassetti $(\bowtie) \cdot$ D. R. Giacobbe $(\bowtie)$.

M. Mikulska $\cdot$ A. Vena

Infectious Diseases Unit, Ospedale Policlinico San

Martino, IRCCS, L.go R. Benzi, 10, 16132 Genoa,

Italy

e-mail: matteo.bassetti@unige.it

D. R. Giacobbe

e-mail: danieleroberto.giacobbe@unige.it

M. Bassetti - D. R. Giacobbe · M. Mikulska

Department of Health Sciences (DISSAL), University

of Genoa, Genoa, Italy

P. Bruzzi

Clinical Epidemiology Unit, Ospedale Policlinico

San Martino, IRCCS, Genoa, Italy

E. Barisione $\cdot$ E. Tagliabue

Interventional Pulmonology, Ospedale Policlinico

San Martino, IRCCS, Genoa, Italy

S. Centanni

Respiratory Unit, ASST Santi Paolo e Carlo, Milan, and Department of Health Sciences, Università degli Studi di Milano, Milan, Italy
Dear Editor,

We thank Manciulli and colleagues for their comment on the recently released guidelines from the Italian Society of Anti-Infective

N. Castaldo $\cdot$ E. Sozio $\cdot$ C. Tascini

Infectious Diseases Clinic, Santa Maria Misericordia Hospital, Udine, Italy

S. Corcione - F. G. De Rosa

Department of Medical Sciences, Infectious

Diseases, University of Turin, Turin, Italy

S. Corcione

Tufts University School of Medicine, Boston, MA, USA

F. Di Marco

Respiratory Unit, Department of Health Sciences, ASST Papa Giovanni XXIII Hospital, University of Milan, Bergamo, Italy

A. Gori · A. Lombardi Infectious Diseases Unit, Fondazione IRCCS $\mathrm{Ca}^{\prime}$ Granda Ospedale Maggiore Policlinico, Milan, Italy

A. Gori · A. Gramegna · A. Lombardi · F. Blasi Department of Pathophysiology and

Transplantation, Università degli Studi di Milano, Milan, Italy 
Therapy (SITA) and the Italian Society of Pulmonology (SIP) on the clinical management of adult patients with coronavirus disease 2019 (COVID-19) outside intensive care units [1, 2]. In their comment, Manciulli and colleagues highlight that, while in the guidelines there is a recommendation against the use of neutralizing monoclonal antibodies in inpatients with COVID-19 (pending results of ongoing trials), the Italian Medicine Agency (AIFA) has recently allowed the use of casirivimab/imdevimab at high dosage in hospitalized seronegative patients with COVID-19 [3]. This apparent discrepancy is mostly related to the fact that the randomized trial (currently available as a nonpeer-reviewed pre-print manuscript [4]) supporting the AIFA decision became available only very recently, after the last literature update dictating guidelines development. The possibility of novel evidence becoming available after the release of the guidelines was not unexpected. Indeed, a novel rigorous literature search supporting a predefined update of the

A. Gori

Centre for Multidisciplinary Research in Health

Science (MACH), University of Milan, Milan, Italy

A. Gramegna · F. Blasi

Internal Medicine Department, Respiratory Unit and Cystic Fibrosis Adult Center, Fondazione IRCCS $\mathrm{Ca}^{\prime}$ Granda Ospedale Maggiore Policlinico, Milan, Italy

G. Granata

Clinical and Research Department for Infectious Diseases, National Institute for Infectious Diseases L. Spallanzani, IRCCS, Rome, Italy

A. Gratarola

Department of Emergency and Urgency, San

Martino Policlinico Hospital, IRCCS, Genoa, Italy

A. E. Maraolo

First Division of Infectious Diseases, Cotugno

Hospital, AORN dei Colli, Naples, Italy

F. Pea $\cdot$ P. Viale

Department of Medical and Surgical Sciences, Alma

Mater Studiorum-University of Bologna, Bologna, Italy

F. Pea

SSD Clinical Pharmacology Unit, University

Hospital, IRCCS Azienda Ospedaliero Universitaria

Di Bologna, Bologna, Italy current guidelines is about to start in November 2021, as stated in the guideline methods [1].

Nonetheless, it is certainly true that novel evidence cannot be ignored. For this reason, pending the predefined update of the current guidelines, we invite Italian physicians to follow the most recent regulatory document, i.e., the current AIFA recommendations on the use of neutralizing monoclonal antibodies in nonhospitalized and hospitalized patients with COVID-19. However, an important point of caution is that neither the certainty of evidence nor the strength for this recommendation can be reliably defined without the proper methodology. Indeed, the formulation of the final, updated SITA and SIP recommendations on the use of neutralizing monoclonal antibodies (both in inpatients and in outpatients and both for intravenous and for other available formulations) will be provided after following the same rigorous systematic steps that were taken during the preparation of the first released document. This is necessary to guarantee the

\author{
N. Petrosillo \\ Infection Control and Infectious Disease Service, \\ University Hospital "Campus-Biomedico", Rome, \\ Italy \\ D. Radovanovic $\cdot$ P. Santus \\ Division of Respiratory Diseases, Ospedale L. Sacco, \\ ASST Fatebenefratelli-Sacco, Milan, Italy \\ P. Santus \\ Department of Biomedical and Clinical Sciences \\ (DIBIC), Università degli Studi di Milano, Milan, \\ Italy
}

A. Signori

Section of Biostatistics, Department of Health

Sciences, University of Genoa, Genoa, Italy

C. Vancheri

Regional Referral Centre for Rare Lung DiseasesUniversity Hospital "Policlinico G. Rodolico", Catania, Italy

C. Vancheri

Department of Clinical and Experimental Medicine, University of Catania, Catania, Italy

P. Viale

Infectious Diseases Unit, University Hospital IRCCS Policlinico Sant'Orsola, Bologna, Italy 
required high-quality standards for guideline development.

\section{ACKNOWLEDGEMENTS}

Funding. No funding or sponsorship was received for this study or publication of this article.

Authorship. All named authors meet the International Committee of Medical Journal Editors (ICMJE) criteria for authorship for this article, take responsibility for the integrity of the work as a whole, and have given their approval for this version to be published.

Author Contributions. Matteo Bassetti: project chair for SITA, project concept, voting panel member, revision of final manuscript and supplementary material. Francesco Blasi: project chair for SIP, project concept, voting panel member, revision of final manuscript and supplementary material. Daniele Roberto Giacobbe: project coordinator, methodology and systematic reviews, assessment of evidence with the GRADE system, drafting of recommendations, drafting of final manuscript and supplementary material, supervision of voting process. Pierluigi Viale, Malgorzata Mikulska, Nicola Petrosillo, Andrea Gori, Carlo Tascini, Francesco Giuseppe De Rosa, Pierachille Santus, Fabiano Di Marco, Stefano Centanni, Carlo Vancheri, Angelo Gratarola, Federico Pea: voting panel members, revision of final manuscript and supplementary material. Antonio Vena, Guido Granata, Silvia Corcione, Emanuela Sozio, Nadia Castaldo, Andrea Lombardi, Andrea Gramegna, Dejan Radovanovic, Elena Tagliabue: search strings development, conduction of systematic reviews, drafting of recommendations, revision of final manuscript and supplementary material. Alessio Signori: development and supervision of methodology together with the project coordinator, revision of final manuscript and supplementary material. Alberto Enrico Maraolo: development and supervision of methodology together with the project coordinator, assessment of evidence with the GRADE system, search strings development, revision of final manuscript and supplementary material. Paolo Bruzzi: development and supervision of methodology together with the project coordinator, assessment of evidence with the GRADE system, revision of final manuscript and supplementary material.

List of Investigators. Matteo Bassetti, Daniele Roberto Giacobbe, Paolo Bruzzi, Emanuela Barisione, Stefano Centanni, Nadia Castaldo, Silvia Corcione, Francesco Giuseppe De Rosa, Fabiano Di Marco, Andrea Gori, Andrea Gramegna, Guido Granata, Angelo Gratarola, Alberto Enrico Maraolo, Malgorzata Mikulska, Andrea Lombardi, Federico Pea, Nicola Petrosillo, Dejan Radovanovic, Pierachille Santus, Alessio Signori, Emanuela Sozio, Elena Tagliabue, Carlo Tascini, Carlo Vancheri, Antonio Vena, Pierluigi Viale, Francesco Blasi.

Disclosure. Outside the submitted work, Daniele Roberto Giacobbe reports an unconditional grant from Correvio Italia and a grant for his institution by Pfizer Inc. Outside the submitted work, Matteo Bassetti has received funding for scientific advisory boards, travel and speaker honoraria from Angelini, Astellas, Bayer, BioMérieux, Cidara, Cipla, Gilead, Menarini, MSD, Pfizer, Shionogi, Tetraphase, Nabriva. Outside the submitted work, Francesco Blasi reports grants and personal fees from AstraZeneca, grants from Bayer, grants and personal fees from Chiesi, grants and personal fees from GlaxoSmithKline, personal fees from Grifols, personal fees from Guidotti, personal fees from Insmed, grants and personal fees from Menarini, personal fees from Novartis, grants and personal fees from Pfizer, personal fees from Zambon, and personal fees from Vertex. Outside the submitted work, Emanuela Barisione reports personal fees from Boehringer Ingelheim, personal fees from Chiesi, and personal fees from GlaxoSmithKline. Outside the submitted work, Federico Pea participated in speaker bureau for Angelini, Basilea Pharmaceutica, Gilead, Hikma, Merck Sharp \& Dohme, Nordic Pharma, Pfzer and Sanofi Aventis, and in advisory board for Angelini, Basilea Pharmaceutica, Correvio, Gilead, Hikma, Merck Sharp 
\& Dohme, Nordic Pharma, Novartis, Pfizer, Shionogi and Thermo-Fisher. Outside the submitted work, Nicola Petrosillo reports personal fees from MSD, personal fees from Pfizer, personal fees from Johnson \& Johnson, personal fees from Shionogi, personal fees from Takeda, and personal fees from Becton \& Dickinson. Outside the submitted work, Pierachille Santus reports personal fees from Gilead. Paolo Bruzzi, Stefano Centanni, Nadia Castaldo, Silvia Corcione, Francesco Giuseppe De Rosa, Fabiano Di Marco, Andrea Gori, Andrea Gramegna, Guido Granata, Angelo Gratarola, Alberto Enrico Maraolo, Malgorzata Mikulska, Andrea Lombardi, Dejan Radovanovic, Alessio Signori, Emanuela Sozio, Elena Tagliabue, Carlo Tascini, Carlo Vancheri, Antonio Vena, and Pierluigi Viale have nothing to disclose.

Compliance with Ethics Guidelines. This article is based on previously conducted studies and does not contain any new studies with human participants or animals performed by any of the authors.

Open Access. This article is licensed under a Creative Commons Attribution-NonCommercial 4.0 International License, which permits any non-commercial use, sharing, adaptation, distribution and reproduction in any medium or format, as long as you give appropriate credit to the original author(s) and the source, provide a link to the Creative Commons licence, and indicate if changes were made. The images or other third party material in this article are included in the article's Creative Commons licence, unless indicated otherwise in a credit line to the material. If material is not included in the article's Creative Commons licence and your intended use is not permitted by statutory regulation or exceeds the permitted use, you will need to obtain permission directly from the copyright holder. To view a copy of this licence, visit http://creativecommons.org/licenses/by$\mathrm{nc} / 4.0 /$.

\section{REFERENCES}

1. Bassetti M, Giacobbe DR, Bruzzi P, et al. Clinical management of adult patients with COVID-19 outside intensive care units: guidelines from the Italian Society of Anti-Infective Therapy (SITA) and the Italian Society of Pulmonology (SIP). Infect Dis Ther. 2021;10(4):1837-85.

2. Manciulli T, Spinicci M, Bartoloni A, et al. Monoclonal antibodies for hospitalized patients with COVID-19 in Italy. Infect Dis Ther. 2021.

3. AIFA. DETERMINA 4 agosto 2021. https://www.aifa. gov.it/documents/20142/847786/GU_187_06_08_ 2021_casirivimab-imdevimab.pdf. Accessed 15 Oct 2021.

4. RECOVERY Collaborative Group, Horby PW, Mafham $\mathrm{M}$, et al. Casirivimab and imdevimab in patients admitted to hospital with COVID-19 (RECOVERY): a randomised, controlled, open-label, platform trial. medRxiv. 2021:2021.06.15.21258542. https://doi. org/10.1101/2021.06.15.21258542.

\section{Publisher's Note}

Springer Nature remains neutral with regard to jurisdictional claims in published maps and institutional affiliations. 